\title{
Development of DAQ to DCS communication in the ATLAS Inner Tracker
}

\author{
Alessandra Palazzo ${ }^{a, *}$ on behalf of the ATLAS Collaboration \\ ${ }^{a}$ INFN Lecce and Università del Salento, Dipartimento di Matematica e Fisica \\ E-mail: alessandra.palazzo@cern.ch
}

The increase of luminosity foreseen for the High-Luminosity LHC phase requires the substitution of the ATLAS Inner Detector with a new tracking detector, called Inner Tracker. It will be an all-silicon system consisting of a pixel and a strip subdetector. The ATLAS wide Front-End Link eXchange system will be the off-detector interface of the data acquisition system to the Inner Tracker. In order to efficiently bring the Inner Tracker into operation, the intercommunication between the data acquisition system and the detector control system is foreseen. Such communication is mediated by open platform communications servers that interface to the different hardware and software resources and to the Finite State Machine supervising all subdetectors. This framework is designed to be flexible, so that it can easily incorporate heterogeneous resources coming from different subsystems, including the Front-End Link eXchange setups. The current status of the implementation of open platform communications servers for the intercommunication between the data acquisition system and the detector control system is described.

The Ninth Annual Conference on Large Hadron Collider Physics - LHCP2021

7-12 June 2021

Online

${ }^{*}$ Speaker 


\section{Inner Tracker for High-Luminosity LHC}

Starting from 2027 the instantaneous luminosity of the Large Hadron Collider (LHC) will reach a peak value of $\mathcal{L}=7.5 \times 10^{34} \mathrm{~cm}^{-2} \mathrm{~s}^{-1}$; this value is higher than the one expected for Run 3 (2022 - 2024) by a factor 3.5. The goal is to achieve an integrated luminosity of $L=4000 \mathrm{fb}^{-1}$ in $\sim 12$ years of operation. This accelerator phase is referred to as High-Luminosity LHC (HL - LHC).

The luminosity increase will cause an increase of the pile-up by a factor 10 with respect to the one observed during Run 2 (2015 - 2018). The current Inner Detector of the ATLAS experiment [1] would not be able to cope with this extremely harsh radiation environment. It will thus be replaced by a new all-silicon detector called Inner Tracker (ITk), whose sketch is shown in Fig. 1. The inner section will feature pixel detectors [3], while in the outer one strip detectors [4] will be used. The ITk has been designed to obtain a tracking performance at least comparable with the one reached in Run 2 despite the increase of the pile-up.

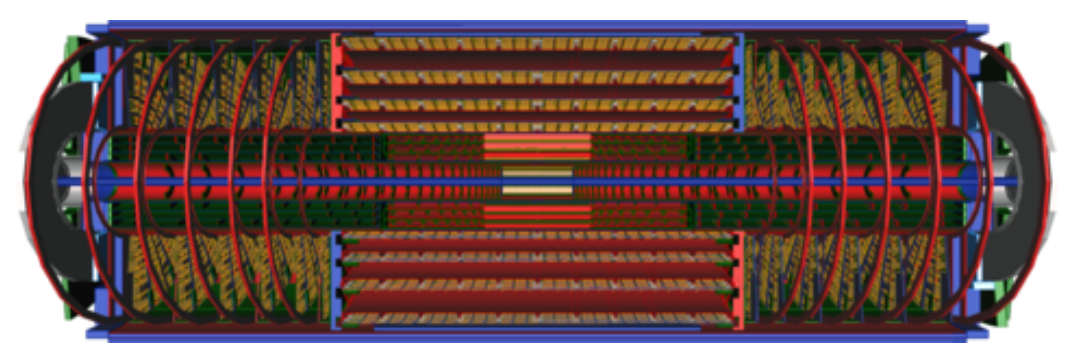

Figure 1: Sketch of the ITk detector. From [2].

\section{The ATLAS Detector Control System}

The Detector Control System (DCS) allows for ATLAS coherent and safe operation. It brings the detector into any desired operational state, continuously monitors and archives the operational parameters, signals any abnormal behavior and allows for manual or automatic corrective actions to be taken [5].

The DCS is divided into Front-End and Back-End. The Front-End includes the detector hardware and its interface, that communicates to the Back-End. The DCS Back-End is formed by different Control Stations interconnected one with another and their interfaces. The top level of the Finite State Machine (FSM) runs over the Global Control Stations, which supervises the overall operation. The FSM provides the abstract representation of the ATLAS DCS. Its top level represents the ATLAS detector, with the sub-detectors and the equipment being the children. They receive commands from the top and propagate status information from the bottom to the higher levels. The communication to different sub-detectors is performed through Supervisory Controls and Data Acquisition (SCADA) applications based on WinCCOA [6].

The communication between the DCS Front-End and Back-End is performed through a middleware layer based on Open Platform Communications Unified Architecture (OPC UA) [7], which is a protocol for intercommunication between different devices, used also for industrial applications. It is based on a client/server model and it is also platform independent, so that it can be easily integrated in different operative systems. 


\section{DAQ to DCS communication}

The ITk Data AcQuisition system (DAQ) needs to communicate to the DCS. This communication can be established through an OPC UA server that can be used for different sub-detectors and connects to the ATLAS Information Service (IS) [9]. The IS is an online software component of the ATLAS Trigger and Data Acquisition (TDAQ) system used to share information between applications in a distributed environment.

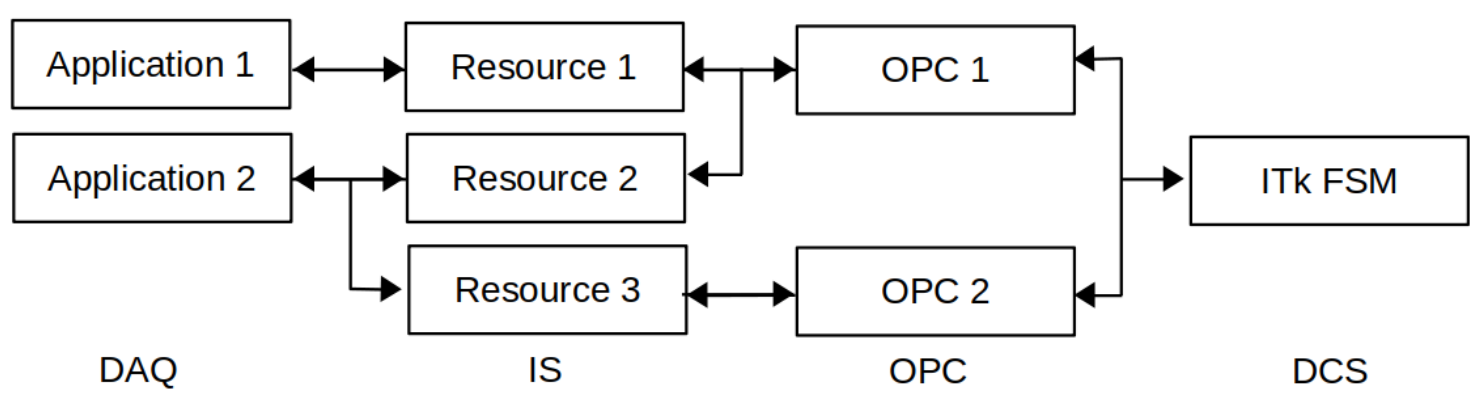

Figure 2: Prototype of the DAQ to DCS communication system foreseen for ITk.

For the sake of this paper, a sketch of the communication system under development for ITk is shown in Fig. 2. The OPC UA servers that interact with the IS can easily be generated through the quick OPC UA server generation framework (Quasar) [8]. They can read from IS or write to it a predefined set of information; for example, the DAQ writes to the IS the status of the run and reads from the DCS the status of the powering of the modules. Different applications can write variables in the IS, and an application can also handle different resources. Several OPC servers can be built and each of them will listen to a certain set of variables. These OPC servers can then be combined into one tree, which implements the interfaces of the DCS to the ITk FSM. The overall system is distributed: each tool (DAQ, IS, OPC servers, WinCCOA) can run on a separate computer.

First steps towards the implementation of the communication system in Fig. 2 have already been taken. An IS object that can be written to the IS or read from it has been created. An OPC server that can read ITk resources from the IS or write them to it has been generated using Quasar. Two example tools have also been created: the first one writes variables on IS address, the second one reads the state of these resources from the IS. In both cases the IS address is specified by the user. Finally, the communication of real power supplies to the IS has been tested.

\section{Summary}

The upgrade of LHC to HL - LHC requires the replacement of the ATLAS Inner Detector with the Inner Tracker detector. It will be built entirely in silicon and will be divided into a pixel and a strip sub-detectors.

A new communication system between the ITk DAQ and DCS is proposed. It will allow one to share information between the two systems by publishing it in the ATLAS Information Service. This information will be handled by servers based on the OPC UA protocol, which allows for intercommunication between different devices. The OPC UA servers will be combined into the ITk Finite State Machine, which is built using the commercial software WinCCOA. 


\section{References}

[1] The ATLAS Collaboration, "The ATLAS Experiment at the CERN Large Hadron Collider", JINST 3 S08003 (2008).

[2] The ATLAS Collaboration, "Expected tracking and related performance with the updated ATLAS Inner Tracker layout at the High-Luminosity LHC", ATL-PHYS-PUB-2021-024 (2021).

[3] The ATLAS Collaboration, "Technical Design Report for the ATLAS Inner Tracker Pixel Detector", ATLAS-TDR-030 (2018).

[4] The ATLAS Collaboration, "Technical Design Report for the ATLAS Inner Tracker Strip Detector", ATLAS-TDR-025 (2018).

[5] The ATLAS Collaboration, "Technical Design Report for the Phase-II Upgrade of the ATLAS Trigger and Data Acquisition System", ATLAS-TDR-029 (2018).

[6] SIMATIC WinCC Open Architecture Portal, "SIMATIC WinCC Open Architecture", https://www.winccoa.com/product-information.html.

[7] The OPC Foundation, "OPC Unified Architecture", https://opcfoundation.org/about/opctechnologies/opc-ua/.

[8] P. P. Nikiel et al., "Quasar - a generic framework for rapid development of OPC UA servers", 15th Int. Conf. on Acc. and Large Exp. Physics Control Systems (ICALEPCS2015) (2015).

[9] S. Kolos, "Information Service - ATLAS TDAQ, version 1.4", https://atlas-tdaqmonitoring.web.cern.ch/IS/doc/userguide/is-usersguide.pdf (2011). 